\title{
Perspectives
}

\section{The usefulness and significance of assessing rapidly progressive spermatozoa}

\author{
Lars Björndahl ${ }^{1,2}$ \\ ${ }^{l}$ Centre for Andrology and Sexual Medicine, Clinic of Endocrinology, Karolinska University Hospital, Huddinge M52, \\ Stockholm, Sweden \\ ${ }^{2}$ Department of Medicine, Karolinska Institutet, Huddinge S-141 86, Stockholm, Sweden
}

\begin{abstract}
It is possible and clinically relevant to distinguish between slow and rapid progressive spermatozoa in basic semen analysis. This is discussed in light of the different purposes of semen analysis for the subfertile couple and the male patient. The two groups of progressive spermatozoa should be distinguished to help ensure that pertinent information available in the semen sample is not neglected.
\end{abstract}

Asian Journal of Andrology (2010) 12: 33-35. doi: 10.1038/aja.2008.50.

Keywords: male factor infertility, manual sperm motility assessment, quality control, rapidly progressive spermatozoa, reference values, semen analysis, staff training

\section{Background}

One commonly recurring problem relevant to the usefulness and significance of semen analysis concerns confusion about different purposes of the analysis. Semen analysis can be used to answer basic short-term questions such as "Can spermatozoa from this man be used to fertilize an oocyte?" or "Can this man's spermatozoa successfully make his partner pregnant?". Adopting a wider scope would allow additional questions to be addressed, such as "Do the reproductive organs of this patient function properly?". With respect to the latter perspective, the investigation and treatment focus not on the symptom (i.e., subfertility) but on any potential underlying disorder. In addition to the possibility that the "male factor" for subfertility could be reduced or even eliminated, the male patient could also benefit from the discovery of potentially

Correspondence to: Dr Lars Björndahl, Centre for Andrology and Sexual Medicine, Clinic of Endocrinology, Karolinska University Hospital, Huddinge M52, Stockholm, Sweden, and Department of Medicine, Karolinska Institutet, Huddinge S-141 86, Stockholm, Sweden.

Fax: +46-8731-0334

Received: 1 November 2008

Accepted: 18 November 2008 life-threatening diseases or disorders that adversely affect quality of life [1]. Another purpose of semen analysis is to generate markers for male fertility in population-based epidemiological and toxicological investigations. In these investigations, the fundamental problem is that results from semen analysis are in general very poor markers for true male fertility.

The results of sperm motility assessment can be used more directly to address problems affecting the man and his reproductive organs. The presence of spermatozoa with substantial forward (progressive) motility speaks against severe infection and inflammatory reactions in the reproductive tract. The presence of inflammatory cells (often peroxidase-positive granulocytes) suggests an ongoing inflammatory reaction and necessitates physical examination and consideration of antibiotic treatment. Spermatozoa exposed to seminal vesicular fluid show decreased motility [2], survival [3] and protection of the sperm chromatin [4], indicating that an abnormal sequence of ejaculation can cause decreased sperm function [5]. Reduced sperm motility can therefore be a symptom of disorders related to male accessory sex gland secretion and to the sequential emptying of these glands. Also in these cases, it is essential for the man's well-being to perform a physical examination and look for possible 
acute inflammatory reactions that may require treatment. Only by examination of a split ejaculate can one discover a disturbed sequence of ejaculation due to previous inflammation in the male reproductive tract [5].

Other uses of sperm motility results are primarily focused on the likelihood of success with assisted reproductive technologies (ART). Of course, there is a general problem in that the ultimate success of ART depends not only on semen analysis but also on factors that are not directly linked to semen analysis, i.e., female factors. Although the influence of female factors, as they are currently understood, can to a certain extent be eliminated in prospective studies, they will remain an issue when the results of semen analysis are appraised as part of the investigation of an individual couple. Therefore, endpoints other than the "take-home-baby rate" are of interest in evaluating the usefulness of semen analysis. With in vitro techniques, fertilization and zygote/embryo cleavage rates are obvious measures. With in vivo fertilization, sperm-cervical mucus penetration tests and rapid progressive sperm motility have long been known to be useful indicators [6-9]. Irrespective of whether one adopts an in vitro or in vivo perspective, progressive sperm motility is thus required for success (with the exception of treatment by intracytoplasmic sperm injection [ICSI]).

\section{Discussion}

The efficient passage of spermatozoa through cervical mucus is dependent on rapid progressive motility [10], i.e., spermatozoa with a forward progression of at least $25 \mu \mathrm{m} \mathrm{s}^{-1}$ $[9,11]$. There are, of course, some differences between sperm velocity assessed with CASA and manually assessed proportions of motile spermatozoa. Although CASA is very accurate for determining the details of sperm motility patterns ("kinematics") and for assessing the paths and propagation speed defined in different ways, manual assessment of semen is much more accurate in discerning between debris, crystals and immotile, dead sperm heads. Therefore, manually assessed sperm concentrations and number of immotile spermatozoa are much more reliable than corresponding data obtained by CASA, provided that the individual performing the manual analysis has been properly trained, and that appropriate internal and external quality control measures are employed $[12,13]$. Those with personal experience in performing andrology laboratory investigations have repeatedly seen that progressive spermatozoa, both slow and rapid, vary widely with respect to various characteristics and abilities to move forward. It is therefore logical to distinguish between those with better progressive motility and those with poor progressive motility (and hence limited functional potential) in basic semen analysis.

Taking into account that manual methods are not as easily calibrated as equipment such as spectrophotometers, it could be argued that a set limit between two categories, for an entity that is continuous, such as sperm motility, is not adequate. However, this is not true. In modern laboratory medicine it is quite common to assign specific, seemingly arbitrary, limits in order to classify individuals, objects, tissues or cells. Sperm morphology assessment is one example of classification as normal or abnormal based on set criteria for characteristics measured on continuous scales (e.g., head size and shape, acrosome size, proportion of the head occupied by vacuoles, midpiece size and contour, tail length, and size of the cytoplasmic residue). It is therefore reasonable to use data obtained by objective measurements to establish a cutoff point between the categories of slow and rapid progressive spermatozoa; $25 \mu \mathrm{m} \mathrm{s}^{-1}[14]$ is based on results derived from analysis of sperm kinematics [7, 11]. It is less important whether the limit is 23 or $25 \mu \mathrm{m} \mathrm{s}^{-1}$ than that the presence of rapidly forward moving spermatozoa is assessed separately from spermatozoa with less progressive motility. In earlier editions of the World Health Organization (WHO) manual on sperm analysis, a reference limit of $25 \%$ was suggested for the proportion of rapid progressive spermatozoa $[14,15]$. In the most recent of these editions, there was also an ill-founded suggestion for assessments to be done at "room temperature". A more recent reference limit of $15 \%$, based on assessment of approximately 100 recent fathers (and corresponding to $95 \%$ of a reference population), was published by Haugen and coworkers [16]. This study used laboratory methods complying with WHO [14] guidelines and the standards of the Nordic Association for Andrology (NAFA) and the European Society of Human Reproduction and Embryology (ESHRE) [17].

Another objection that has sometimes been raised against distinguishing between slow and rapid spermatozoa is that it is not feasible when doing manual analysis. However, in laboratories with standardized, systematic training and functional quality control, this procedure has been performed routinely for many years $[12,18,19]$. In the 4-day courses on basic semen analysis developed and given by the Special Interest Group in Andrology of the ESHRE, the immediate effects of standardized training are obvious [13]. The level of training and the quality assurance measures used do not differ significantly from those required for any competent laboratory performing semen analysis according to the recommendations in the WHO manual [14].

The final issue to address is whether making the distinction between slow and rapid progressive spermatozoa has any clinical importance. Verheyen and coworkers in Brussels [20] found that no or very few spermatozoa with grade "a" motility indicated a high risk of complete fertilization failure with conventional IVF. Sifer and coworkers in France [21] found that grade "a" motility together with sperm-zona pellucida binding tests were the most useful measures with 
which to predict fertilization failures with IVF. Note that the predictive power here is not for success (e.g. "fertility") but for predicting failure in treatment, an outcome that is actually more important when making decisions regarding a couple's treatment options. Thus, making the distinction between slow and rapid progressive spermatozoa is of great clinical relevance and utility.

\section{Conclusion}

Distinguishing between slow and rapid progressive spermatozoa is essential, and it is possible to standardize procedures to accomplish this in manual assessments in any routine semen analysis laboratory. To neglect the distinction between the two groups of progressive spermatozoa is actively to ignore information that is available in the semen sample, and to omit such information would impoverish the clinical usefulness of semen analysis.

\section{References}

1 Jequier AM. The importance of diagnosis in the clinical management of infertility in the male. Reprod Biomed Online 2006; 13: 331-5.

2 Lindholmer C. The importance of seminal plasma for human sperm motility. Biol Reprod 1974; 10: 533-42.

3 Lindholmer C. Survival of human spermatozoa in different fractions of split ejaculate. Fertil Steril 1973; 24: 521-6.

4 Björndahl L, Kjellberg S, Kvist U. Ejaculatory sequence in men with low sperm chromatin-zinc. Int J Androl 1991; 14: 174-8.

5 Björndahl L, Kvist U. Sequence of ejaculation affects the spermatozoon as a carrier and its message. Reprod Biomed Online 2003; 7: 440-8.

6 Aitken RJ, Sutton M, Warner P, Richardson DW. Relationship between the movement characteristics of human spermatozoa and their ability to penetrate cervical mucus and zona-free hamster oocytes. J Reprod Fertil 1985; 73: 441-9.

7 Mortimer D, Pandya IJ, Sawers RS. Relationship between human sperm motility characteristics and sperm penetration into human cervical mucus in vitro. J Reprod Fertil 1986; 78: 93-102.
8 Aitken RJ, Warner PE, Reid C. Factors influencing the success of sperm-cervical mucus interaction in patients exhibiting unexplained infertility. J Androl 1986; 7: 3-10.

9 Hirano Y, Shibahara H, Obara H, Suzuki T, Takamizawa S, et al. Relationships between sperm motility characteristics assessed by the computer-aided sperm analysis (CASA) and fertilization rates in vitro. J Assist Reprod Genet 2001; 18: 213-8.

10 Keel BA, Webster BW. Correlation of human sperm motility characteristics with an in vitro cervical mucus penetration test. Fertil Steril 1988; 49: 138-43.

11 Mortimer ST. A critical review of the physiological importance and analysis of sperm movement in mammals. Hum Reprod Update 1997; 3: 403-39.

12 Mortimer D, Shu MA, Tan R. Standardization and quality control of sperm concentration and sperm motility counts in semen analysis. Hum Reprod 1986; 1: 299-303.

13 Björndahl L, Barratt CL, Fraser LR, Kvist U, Mortimer D. ESHRE basic semen analysis courses 1995-1999: immediate beneficial effects of standardized training. Hum Reprod 2002; 17: 1299-305.

14 World Health Organization. WHO Laboratory Manual for the Examination of Human Semen and Sperm-Cervical Mucus Interactions, 4th edn. Cambridge: Cambridge University Press; 1999.

15 World Health Organization. WHO Laboratory Manual for the Examination of Human Semen and Sperm-Cervical Mucus Interactions, 3rd edn. Cambridge: Cambridge University Press; 1992.

16 Haugen TB, Egeland T, Magnus O. Semen parameters in Norwegian fertile men. J Androl 2006; 27: 66-71.

17 Kvist U, Björndahl L, editors. Manual on Basic Semen Analysis. Oxford: Oxford University Press; 2002.

18 Mortimer D. Laboratory standards in routine clinical andrology. Reprod Med Rev 1994; 3: 97-111.

19 Mortimer D. Practical Laboratory Andrology. Oxford: Oxford University Press; 1994.

20 Verheyen G, Tournaye H, Staessen C, De Vos A, Vandervorst $\mathrm{M}$, et al. Controlled comparison of conventional in-vitro fertilization and intracytoplasmic sperm injection in patients with asthenozoospermia. Hum Reprod 1999; 14: 2313-9.

21 Sifer C, Sasportes T, Barraud V, Poncelet C, Rudant J, et al. World Health Organization grade 'a' motility and zona-binding test accurately predict IVF outcome for mild male factor and unexplained infertilities. Hum Reprod 2005; 20: 2769-75. 